\title{
Pathophysiological Studies Are Mandatory to Understand the Benefit of Proton Pump Inhibitors in Patients with Idiopathic Pulmonary Fibrosis
}

TO THE EDITOR: We read with great interest the paper by Lee et $\mathrm{al}^{1}$ on the protective effect of proton pump inhibitors (PPIs) for survival in patients with gastroesophageal reflux disease (GERD) and idiopathic pulmonary fibrosis (IPF). The Authors reviewed a very large number of consecutive adult patients with IPF ( $\mathrm{n}=$ 786) and concluded that the prevalence of GERD is lower in Korean patients than in other countries and that PPI use for at least 4 months may have a protective effect against IPF-related mortality.

Although these data appear interesting, relevant limitations of this study were its retrospective nature and the fact that the diagnosis of GERD was not based on sound methods, in that only 18 out of 107 patients had erosive esophagitis at endoscopy, while the remaining ones had non-erosive reflux disease $(n=66)$ or typical reflux symptoms without esophagogastroduodenoscopy ( $\mathrm{n}=$ 23). Moreover, no functional tests were performed (or described) in them. This is particularly relevant since it has been shown in previous series that a large number of IPF patients lack of GERD symptoms despite objective evidence of GERD at endoscopy or reflux monitoring. ${ }^{2-4}$ Indeed, in IPF patients a phenomenon so called "silent reflux" has been described and the consequent microaspiration into the lungs has been mainly implicated in the pathogenesis of this condition. ${ }^{5}$

In contrast with the paper of Lee et al, ${ }^{1}$ many previous studies have reported a higher prevalence of GERD in patients with $\mathrm{IPF}^{2-4}$ and this important relationship has been sustained by pathophysiological investigations which allowed to detect objectively the presence of an abnormal reflux. For instance, Lee et al ${ }^{1}$ did not mention a study in IPF individuals performed with 24-hour impedance $\mathrm{pH}$ monitoring, which is nowadays considered to be the best test for measuring gastroesophageal reflux, also in case of atypical manifestations. ${ }^{6-8}$ In this study, patients with IPF had significantly higher esophageal acid exposure and greater number of acid refluxes than controls, but also weakly acidic refluxes were remarkably increased. ${ }^{9}$ Furthermore, more bile acids and pepsin were detected in both broncheoalveolar lavage fluid and saliva as strong confirmation of the risk of gastric aspiration in upper airways of these patients. Similarly, in an earlier pathophysiological study in a group of scleroderma patients with various degrees of pulmonary fibrosis based on high resolution computed tomography, not only acid but also weakly acidic refluxes were found much higher in patients with more severe than in those with mild or moderate pulmonary fibrosis. ${ }^{10}$

In conclusion, we would like to emphasize that reflux monitoring plays a fundamental role in confirming the presence of GERD in IPF patients and, therefore, the benefit of PPI use in them should be evaluated in carefully investigated subjects in order to provide stronger evidence that PPI may be helpful for their wellbeing and survival.

\section{Edoardo Savarino, ${ }^{1}$ Patrizia Zentilin, ${ }^{2}$ Elisa Marabotto, ${ }^{2}$ and Vincenzo Savarino ${ }^{2}$ 'Gastroenterology Unit, Department of Surgery, Oncology and Gastroenterology, University of Padua, Padua, Italy; and ${ }^{2}$ Gastroenterology Unit, Department of Internal Medicine, University of Genoa, Genoa, Italy}

1. Lee CM, Lee DH, Ahn BK, et al. Protective effect of proton pump inhibitor for survival in patients with gastroesophageal reflux disease and idiopathic pulmonary fibrosis. J Neurogastroenterol Motil 2016;22:444451.

2. Raghu G, Freudenberger TD, Yang S, et al. High prevalence of abnormal acid gastro-esophageal reflux in idiopathic pulmonary fibrosis. Eur Resp J 2006;27:136-142.

3. Salvioli B, Belmonte G, Stanghellini V, et al. Gastroesophageal reflux and interstitial lung disease. Dig Liver Dis 2006;38:879-884.

4. Tobin RW, Pope CE 2nd, Pellegrini CA, Emond MJ, Sillery J, Raghu G. Increased prevalence of gastroesophageal reflux in patients with idiopathic pulmonary fibrosis. Am J Respir Crit Care Med 1998;158:1804-1808. 
5. Raghu G, Meyer KC. Silent gastroesophageal reflux and microaspiration in IPF: mounting evidence for anti-reflux therapy? Eur Respir J 2012;39:242-245.

6. Zentilin P, Dulbecco P, Savarino E, Giannini E, Savarino V. Combined multichannel intraluminal impedance and $\mathrm{pH}$-metry: a novel technique to improve detection of gastroesophageal reflux: literature review. Dig Liver Dis 2004;36:565-569.

7. Ribolsi M, Savarino E, De Bortoli N, et al. Reflux pattern and role of impedance-pH variables in predicting PPI response in patients with suspected GERD-related chronic cough. Aliment Pharmacol Ther 2014;40:966-973.

8. de Bortoli N, Nacci A, Savarino E, et al. How many cases of laryngopharyngeal reflux suspected by laryngoscopy are gastroesophageal reflux disease-related? World J Gastroenterol 2012;18:4363-4370.

9. Savarino E, Carbone R, Marabotto E, et al. Gastroesophageal reflux and gastric aspiration in idiopathic pulmonary fibrosis patients. Eur Respir J 2013;42:1322-1331.

10. Savarino V, Bazzica M, Zentilin P, et al. Gastroesophageal reflux and pulmonary fibrosis in scleroderma: a study using $\mathrm{pH}$-impedance monitoring. Am J Respir Crit Care Med 2009;179:408-413.

Conflicts of interest: None.

Author contributions: Edoardo Savarino, Patrizia Zentilin, Elisa Marabotto, and Vincenzo Savar designed the study, wrote the manuscript, and approved the final version. 